\title{
Early determination of toxicant concentration in water supply using MHE
}

\author{
F. Ibrahim ${ }^{*}$ B. Huang* J. Xing ${ }^{* *}$ B. Jayasankar ${ }^{*}$ \\ * Department of Chemical and Material Engineering, University of \\ Albert, Canada T6G2G6. fadi.ibrahim@ualberta.ca, \\ biao.huang@ualberta.ca,jayasank@ualberta.ca \\ ** Department of Laboratory Medicine and Pathology, University of \\ Alberta, Canada T6G 2S2.jzxing@ualberta.ca
}

\begin{abstract}
In this paper, a novel application of state estimation in environmental engineering is presented. Filtering techniques including moving horizon estimator (MHE) and extended Kalman filter (EKF) are used for early concentration estimation of toxic agents existing in water supply. The purpose is to integrate the filtering techniques with an early warning system enabling an early detection of the presence of toxicants in the water supply system and quantifying their concentrations. The estimation is based on dynamic measurements generated by a real-time cell electronic sensor (RT-CES) and cytotoxicity dynamic models.
\end{abstract}

Keywords: state estimation, extended Kalman filter, moving horizon state estimation, cytotoxicity, real-time cell electronic sensor, early warning, water protection.

\section{INTRODUCTION}

Drinking water may be contaminated by a range of chemical, microbial and physical hazards that could pose risks to health if they are present at high levels. Examples of chemical hazards include mercury, chromium, arsenic, etc. The sources of these toxicants differ with respect to the toxicant. Mercury for instance, occurs as a result of both natural (volcanic, forest fires and oceanic releases) and anthropogenic sources (mining, smelting and other industrial activities) in our environment as mentioned by Wang et al. (2004).

The effects of toxicants on the human cells are referred to as cytotoxicity. In other words, cytotoxicity is the characteristic of being toxic to living cells, including cell killing, cell lysis and certain cellular pathological changes, such as cellular morphological change and adhesion change as reported in Xing et al. (2005). Therefore, citizens must be alerted as early as possible when water is contaminated. For this purpose, an early warning system is necessary for detection of any sudden deterioration in the quality of water supply. An efficient detection must include the ability of an early determination of the presence of a toxicant at low concentration. Thus, our main objective in this paper is to use filtering techniques, such as moving horizon estimation (MHE) and extended Kalman filter (EKF) to determine on-line the concentration of such a toxicant in water supply. To achieve this purpose, mathematical modeling and real-time measurements are necessary. Two mathematical models have been developed and validated by Huang and Xing (2006) to predict cell toxicity response to mercury (II) chloride and sodium dichromate [chromium (VI)] toxicity. The measurements of toxicity

\footnotetext{
* The authors gratefully acknowledge the support of the Natural Sciences and Engineering Research Council of Canada (NSERC).
}

response were recorded using Real-Time Cell Electronic Sensor (RT-CES). These two models are able to predict cell responses to different values of toxicant concentration and allow assessment of the biological consequences of toxic chemicals in environmental contamination. In this paper, we reverse the modeling procedure. We are interested in the estimation of toxicant concentration for a given dynamic model through on-line monitoring data sampled from RT-CES. The organization of this paper is as follows. The monitoring procedure and the mathematical models are revisited in Section 2 and Section 3 respectively. The procedure of concentration estimation and the validation results are presented in Section 4 including concentration estimation using both MHE and EKF. Concluding remarks are given in Section 5.

\section{EQUIPMENT AND MONITORING PROCEDURE REVISIT}

1) Equipment: The RT-CES system (ACEA Biosciences, CA, U.S.A.) is used for this study and has been described in (Xing et al., 2005; Huang and Xing, 2006). Briefly, as shown in Fig. 1, it consists of a 16x microelectronic sensor devices having 16 plastic wells in microtiter plate format, a device station and an electronic sensor analyzer. Cells are grown onto the surfaces of microelectronic sensors. In operation, the sensor devices with cultured cells are mounted to a device station placed inside a $\mathrm{CO} 2$ incubator. Electrical cables connect the device station to the sensor analyzer. Under the control of RT-CES software, the sensor analyzer automatically selects wells to be measured and continuously conducts measurements. The electronic impedance can then be transferred to a computer and recorded. A parameter termed cell index $(C I)$ is derived to represent cell status based on the measured electrical impedance. The frequency dependent 
electrode impedance (resistance) without or with cells present in the wells is represented as $R_{b}(f)$ and $R_{\text {cell }}(f)$, respectively. The CI is calculated by:

$$
C I=\max _{i=1, \ldots, n}\left[\frac{R_{\text {cell }}\left(f_{i}\right)}{R_{b}\left(f_{i}\right)}-1\right]
$$

where $n$ is the number of the frequency points at which the impedance is measured. Several features of the $C I$ can be derived: (1) Under the same physiological conditions, if more cells attach onto the electrodes, the larger impedance value leading to a larger CI value will be detected. If no cells are present on the electrodes or if the cells are not well-attached onto the electrodes, $R_{\text {cell }}(f)$ is the same as $R_{b}(f)$, leading to $C I=0 ;(2)$ A large $R_{\text {cell }}(f)$ value leads to a larger $C I$. Thus, $C I$ is a quantitative measure of the number of cells attached to the sensors; (3) For the same number of cells attached to the sensors, changes in cell status, such as morphological change, lead to change of $C I$.

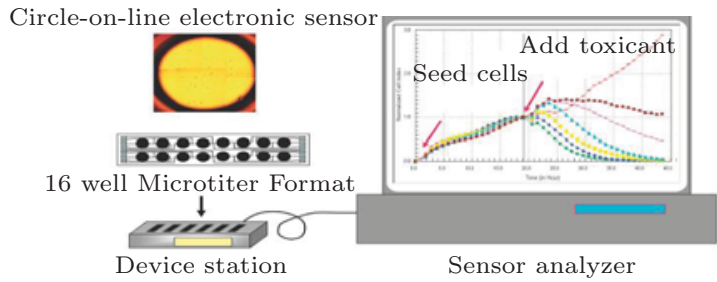

Fig. 1. The Real-Time Cell Electronic Sensor

In addition to cell numbers, the impedance also depends on the extent to which cells attach to the electrodes. For example, if cells spread, there will be a greater cell/electrode contact area, resulting in larger impedance. Thus, the cell biological status including cell viability, cell number, cell morphology and cell adhesion will all affect the measurements of electrode impedance that is reflected by $C I$ on the RT-CES system. Therefore, a dynamic pattern of a given CI curve can indicate sophisticated physiological and pathological responses of the living cells to a given toxic compound (Xing et al., 2005).

2) Dynamic growth with toxicity: Two environmental toxicants, mercury (II) chloride and sodium dichromate [chromium (VI)], were used for cytotoxicity assessment on the 16 sensor device. The cell line NIH 3T3 was tested. The starting cell number was 10000 cells per sensor wells. The cell growth on the sensor device was monitored every hour up to $24 \mathrm{~h}$ in real-time by the RT-CES system. When the $C I$ values reached a range between 1.0 and 1.2 , the cells were then exposed to either mercury (II) chloride, or chromium (VI) at different concentrations. Fig. 2 shows dynamic cytotoxic response to different doses of chromium (VI). Fig. 3 shows dynamic cytotoxic response to mercury (II) chloride. In both cases the cytotoxicity response is dose dependent and increasing dose leads to decreasing $(C I)$.

\section{MATHEMATICAL MODELING}

As mentioned in the introduction, the cytotoxicity mechanism is complex and cell response to toxicity depends on cell type, toxicant type, toxicant concentrations and the time of exposure to the toxicant. In Huang and Xing (2006), two types of models were developed and validated

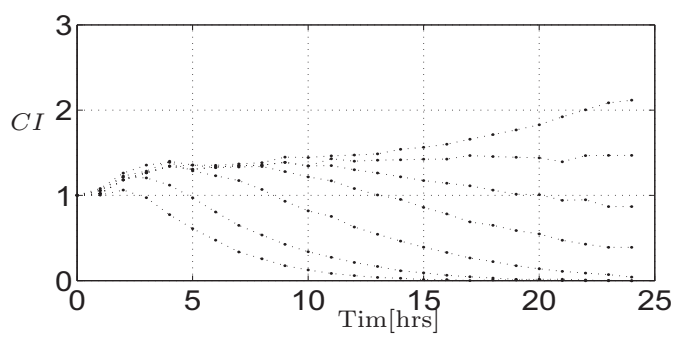

Fig. 2. Dynamic cytotoxic response of NIH 3 T3 cells to different doses of chromium (VI): 0; 0.62; 0.91; 1.97; 2.89; 4.25; 5.78 in the unit of $\mu M$. Increasing dose leads to decreasing $(C I)$.

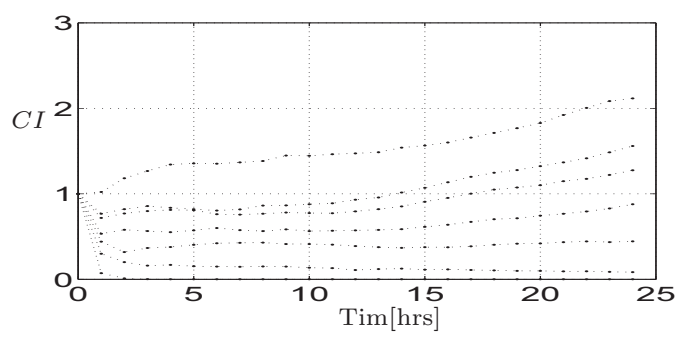

Fig. 3. Dynamic cytotoxic response of NIH 3 T3 cells to different doses of mercury (II): 0; 10.43; 15.2; 22.35; 32.8; 48.3; 71 in the unit of $\mu M$. Increasing dose leads to decreasing (CI).

to predict cell toxicity response to mercury (II) chloride, and sodium dichromate [chromium (VI)] stimulations. In both models, it was suggested that the process of cytotoxicity follows two-step mechanism: (1) uptake of toxicant by cells and (2) killing of the cells. The uptake mechanism describes the transport process of the toxicant into a cell as illustrated in Fig. 4 (Huang and Xing, 2006). This mechanism relates the extracellular concentration $c_{e}$ (representing the concentration of a toxicant in the environment) and the intracellular concentration $c_{i}$ (concentration inside the cell) and it is described by El-Kareh and Secomb (2005) as follows :

$$
\dot{c}_{i}=k_{1}\left(k_{2} c_{e}+\frac{k_{3} c_{e}}{k_{4}+c_{e}}-c_{i}\right)
$$

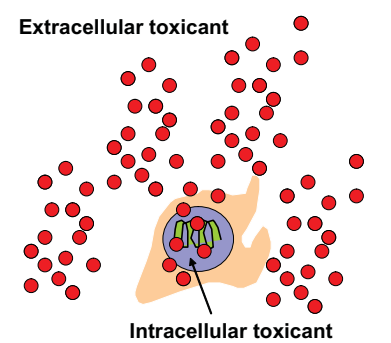

Fig. 4. Schematic of transport process of toxicant onto cell.

The first step in cytotoxicity (the uptake mechanism) is supposed to be rather consistent; however the second step (cell killing) differs with respect to the toxicant and can be described through cell population dynamics as $\dot{N}=f(C, N)$ where $N$ is the cell population and $C$ can be the intracellular or the extracellular concentration of the toxicant or a combination of them. It depends on the type of the toxicant. In the following, we present the mathematical models of cell killing under the effect of two toxicants, mercury (II) chloride, and sodium dichromate [chromium (VI)] as described in Huang and Xing (2006). 


\subsection{Mathematical modeling of [chromium (VI)] toxicity}

The cell exposed to dichromate [chromium (VI)] is killed by apoptosis mechanism which is a highly regulated process and is described as programmed cell death. The apoptosis mechanism mainly depends on the intracellular concentration of the toxicant. This is described by the dynamics of cell pullulation given by Eliaz et al. (2004) as $\dot{N}=N\left(k_{s}-k c_{i}\right)$. As proposed by Huang and Xing (2006), this mechanism yields the following system of differential equations for describing dichromate [chromium (VI)] effects on cells population dynamics:

$$
\begin{aligned}
& \dot{c_{i}}=k_{1}\left(k_{2} c_{e}+\frac{k_{3} c_{e}}{k_{4}+c_{e}}-c_{i}\right) \\
& \dot{N}=N\left(k_{s}-k c_{i}\right)
\end{aligned}
$$

The parameters of this model (3) are estimated from the experiment data and presented in Table 1.

Table 1. Estimated parameters for model (3)

\begin{tabular}{cccccc}
\hline$k_{1}$ & $k_{2}$ & $k_{3}$ & $k_{4}$ & $k_{s}$ & $k$ \\
0.0146 & 2.9399 & 0.0080 & 29.2418 & 0.0425 & 0.1041 \\
\hline
\end{tabular}

\subsection{Mathematical modeling of mercury (II) chloride toxicity}

In Huang and Xing (2006), mercury cytotoxicity has both necrosis mechanism and apoptosis mechanism. The necrosis describes an accidental cell death caused, for example, by chemical or physical assault to the cell which may make cells die by direct disruption of cell membrane. Thus necrosis mechanism mainly depends on extracellular concentration of the toxicant. The cell population dynamics, together with the uptake mechanism expressed by eqn. (2), under mercury (II) chloride toxicity effect is described as follows:

$$
\begin{aligned}
& \dot{c_{i}}=k_{1}\left(k_{2} c_{e}+\frac{k_{3} c_{e}}{k_{4}+c_{e}}-c_{i}\right) \\
& \dot{N}=N\left(k_{5}+k_{6} c_{i}+k_{7} c_{e}\right)
\end{aligned}
$$

The parameters of model (4) are presented in Table 2.

Table 2. Estimated parameters for model (4)

\begin{tabular}{ccccccc}
\hline$k_{1}$ & $k_{2}$ & $k_{3}$ & $k_{4}$ & $k_{5}$ & $k_{6}$ & $k_{7}$ \\
7.735 & 1.108 & 3.21 & 12.8 & 0.0312 & 0.2084 & -0.2364 \\
\hline
\end{tabular}

\section{RAPID TOXICANT CONCENTRATION ESTIMATION}

The on-line estimation of the key parameter (the concentration of toxicant $c_{e}$ ) is critical due to two reasons. First, as being well known the concentration itself is usually not easily measurable due to technical or economical limitations especially for biomedical processes. The key toxicants are usually measured by high performance liquid chromatography (HPLC) and liquid chromatographymass spectrometry (LC-MS) which are expensive equipments. Second, an early determination (detection) of the concentration of such a toxicant is important for an early warning system (which we aim to develop) in order to detect any sudden deterioration in the quality of water supply. Deterioration in water quality mainly means increase of the concentrations (or even the presence) of toxicants. For the early warning system, it is necessary to do on-line estimation of $c_{e}$.

For on-line estimation, several methods for state estimation are available such as EKF and MHE. EKF is a popular state estimation technique and considered as the standard choice for estimating state for nonlinear systems due to lower computation load and more stable property. However, additional physical insights about the process may help in state estimation to prevent negative concentration for instance. This kind of insights can not be considered in EKF. On the other hand, this physical insights can be added as inequality constraints and integrated with an optimal state estimation scheme formulated as a quadratic problem such as MHE (Rao et al., October 2001).

We use mainly MHE for on-line estimation of the key parameter $c_{e}$ and we also use EKF as an alternative (usually a quicker method) of the estimation. This may be considered as a comparison to demonstrate by biological application examples the benefits of using MHE on one hand. On the other hand, the EKF is also imbedded in the MHE and is naturally used for a comparison. The superiority of MHE has also been pointed out by several authors through a number of applications such as in Rao and Rawlings (2002) and Haseltine and Rawlings (2005) for instance.

Before starting the procedure of on-line state estimation of the key parameter (the extracellular concentration $c_{e}$ ) for our biological application, an identifiability test is necessary. We present in the next section an identifiability test for mercury (II) chloride toxicity model (4) for an illustration. An identifiability test for chromium (VI) toxicity model (3) can be performed similarly.

\subsection{Identifiability}

A mathematical model is identifiable if there exist no two parameter sets which have the same input-output behavior. In other words, a model is not identifiable if there exists no unique parameter set to explain the input-output behavior. Identifiability is a pre-analysis for parameter estimation problem to determine the uniqueness of the parameter solution obtained from the estimation process. A number of methods are available for testing identifiability of parametric models. For testing the identifiability of the mercury (II) toxicity model (4), the Taylor series approach is utilized (see Walter and Pronzato (1996)). A brief description of the approach is given below. Consider the following model :

$$
\begin{aligned}
\dot{x}(t) & =f(x(t), u(t), t, p), \quad x(0)=x_{0}(p) \\
y(t, p) & =h(x(t), p)
\end{aligned}
$$

where $p$ is the model parameters set.

If $a_{k}(p)=\lim _{t \longrightarrow 0+} \frac{d^{k}}{d t^{k}} y(t, p)$ then a sufficient condition for model (5) to be uniquely identifiable is :

$$
a_{k}(\hat{p})=a_{k}\left(p^{*}\right), \quad k=0,1, \ldots, k_{\max }, \Longrightarrow \hat{p}=p^{*}
$$

where $k_{\max }$ is a positive integer, small enough for the computations to remain tractable. 
Since mercury (II) toxicity model (4) has only one parameter (the concentration $c_{e}$ ), checking for identifiability reduces to checking for conditions under which the parameter can be observed from the Taylor series coefficients. The first two coefficients of the series for the mercury (II) toxicity model can be determined as:

$$
\begin{aligned}
& a_{0}(p)=N(0) \\
& a_{1}(p)=\dot{N}(0)=N(0)\left(k_{5}+k_{6} c_{i}(0)+k_{7} c_{e}\right)
\end{aligned}
$$

Solving the equation (6) for $c_{e}$ yields,

$$
c_{e}=\frac{k_{6} c_{i}(0) N(0)+k_{5} N(0)-\dot{N}(0)}{-k_{7} N(0)}
$$

Therefore $c_{e}$ is identifiable if:

$$
\begin{aligned}
-k_{7} N(0) & \neq 0 \quad \text { and } \\
k_{6} c_{i}(0) N(0)+k_{5} N(0)-\dot{N}(0) & \neq 0
\end{aligned}
$$

Measurements evolution shown in Fig. 2 and values of the estimated parameters in table 2 satisfy both conditions, equations 7 and 8 .

\subsection{Extended Kalman Filter (EKF) formulation}

Before presenting EKF formulation, considering the problem of estimating the state of system modeled by the nonlinear state space equation :

$$
\begin{aligned}
x_{k+1} & =f\left(x_{k}, u_{k}, k\right)+G w_{k} \quad k=0,1,2 \ldots \\
y_{k} & =g\left(x_{k}, k\right)+v_{k}
\end{aligned}
$$

where, $x_{k} \in \mathbb{R}^{n}$ is the state vector, $y_{k} \in \mathbb{R}^{p}$ the measured output, $w_{k} \in \mathbb{R}^{n}$ state disturbance and $v_{k} \in \mathbb{R}^{p}$ the measurement noise. The EKF linearizes the nonlinear system and then applies the Kalman filter to obtain the state estimation. The method can be summarized in a recursion structure similar to linear Kalman filter for a nonlinear system described above by equations (9) (see Haseltine and Rawlings (2005)):

\section{Prediction step}

$$
\begin{aligned}
& \hat{x}_{k \mid k-1}=f\left(\hat{x}_{k-1 \mid k-1}, u_{k-1}, w_{k-1}\right) \\
& P_{k \mid k-1}=A_{k-1} P_{k-1 \mid k-1} A_{k-1}^{T}+G_{k-1} Q_{k-1} G_{k-1}^{T}
\end{aligned}
$$

Update step

$$
\begin{array}{r}
\hat{x}_{k \mid k}=\hat{x}_{k \mid k-1}+K_{k}\left(y_{k}-g\left(\hat{x}_{k \mid k-1}\right)\right) \\
K_{k}=P_{k \mid k-1} C_{k}^{T}\left[C_{k} P_{k \mid k-1} C_{k}^{T}+R_{k}\right]^{-1} \\
P_{k \mid k}=P_{k \mid k-1}-K_{k} C_{k} P_{k \mid k-1}
\end{array}
$$

in which, the following linearizations are made

$A_{k}=\frac{\partial f\left(x_{k}, u_{k}, k\right)}{\partial x_{k}^{T}}, \quad G_{k}=\frac{\partial f\left(x_{k}, u_{k}, k\right)}{\partial w_{k}^{T}}, \quad C_{k}=\frac{\partial g\left(x_{k}\right)}{\partial x_{k}^{T}}$

\subsection{Moving Horizon State Estimation (MHE) formulation}

The MHE strategy belongs to a class of optimization methods for on-line determination of state. The optimization problem is formulated as a least-squares problem where the decision variables are chosen to minimize the sum of the squared errors between the available measurements and model prediction. When a new measurement becomes available, this optimization is repeated by adding the new measurement to the past measurements each sampling time. This leads to growing computational burden of solving the least-squares optimization, known as full information estimation problem. MHE reduces this computational cost by considering a finite horizon of only the last $N_{h}$ measurements in the optimization problem and information provided by past data beyond the horizon is captured by arrival cost. This optimization is repeated each sampling time by including a new measurement and discarding the first measurement while keeping a fixed horizon length $\left(N_{h}\right)$. In other words, the MHE algorithm is a least square optimization problem solved over a window of fixed horizon length $\left(N_{h}\right)$. This window moves one step ahead each time after solving an optimization problem with a quadratic cost function $\left(\Psi_{k}\right)$ of the following form (for more details see Rao et al. (2003)):

$$
\begin{array}{r}
\min _{\left\{\hat{w}_{k-N_{h}-1 \mid k}, \ldots, \hat{w}_{k-1 \mid k}\right\}} \Psi_{k}: \Psi_{k}=\hat{w}_{k-N_{h}-1 \mid k}^{T} Q_{-N_{h} \mid k}^{-1} \hat{w}_{k-N_{h}-1 \mid k} \\
+\sum_{j=k-N_{h}}^{k-1} \hat{w}_{j \mid k}^{T} Q^{-1} \hat{w}_{j \mid k}+\sum_{j=k-N_{h}}^{k} \hat{v}_{j \mid k}^{T} R^{-1} \hat{v}_{j \mid k}
\end{array}
$$

subject to the state equality constrains:

$$
\begin{aligned}
\hat{x}_{k-N_{h} \mid k} & =\bar{x}_{k-N_{h} \mid k}+\hat{w}_{k-N_{h}-1 \mid k} \\
\text { with } \bar{x}_{k-N_{h} \mid k} & =f\left(\hat{x}_{k-N_{h}-1 \mid k-1}^{*}, u_{k-N_{h}-1}, k\right) \\
\hat{x}_{j+1 \mid k} & =f\left(\hat{x}_{j \mid k}, u_{j}\right)+\hat{w}_{j \mid k}, j=k-N_{h}, \ldots, k-1 \\
y_{j} & =g\left(\hat{x}_{j \mid k}, k\right)+\hat{v}_{j \mid k}, j=k-N_{h}, \ldots, k
\end{aligned}
$$

with the possibility to incorporate inequality constraints on the state, state disturbance and process noise:

$$
\begin{gathered}
w_{\min }<A w_{j}<w_{\max }, \quad x_{\min }<A x_{j}<x_{\max }, \\
v_{\min }<A v_{j}<v_{\max }, \quad j=k-N_{h}-1, \ldots, k-1
\end{gathered}
$$

where $Q$ is the covariance of the state disturbance and $R$ is the covariance of process noise.

The term $\hat{w}_{k-N_{h}-1 \mid k}^{T} Q_{-N_{h} \mid k}^{-1} \hat{w}_{k-N_{h}-1 \mid k}$ approximates the arrival cost which summarizes the effects of the past information before $t=k-N_{h}$. The weighting term $Q_{-N_{h} \mid k}$ initially represents the covariance of the prior state estimate $\bar{x}_{k-N_{h}}$ and is computed according to EKF covariance update formula (Rao et al., 2003):

$$
\begin{gathered}
Q_{-N_{h} \mid k+1}=A_{k} Q_{-N_{h} \mid k} A_{k}^{T}+G_{k} Q_{k} G_{k}^{T}- \\
A_{k} Q_{-N_{h} \mid k} C_{k}^{T}\left[C_{k} Q_{-N_{h} \mid k} C_{k}^{T}+R_{k}\right]^{-1} C_{k} Q_{-N_{h} \mid k} A^{T}
\end{gathered}
$$

where $A_{k}, C_{k}$ and $G_{k}$ result from linearizing the model (9) around the estimated trajectory.

In the full information problem there is no arrival cost because the whole information (all available measurements) is used each sampling time in the optimization while in MHE, only a subset of the information is used and the rest is approximated by the arrival cost. Thus, MHE is an approximation of the full information problem and therefore stability issue arises. The key to preserving 
stability is how to approximately summarize the old data, equivalently, how to find the best approximation of the arrival cost, an explicit expression which rarely exists in nonlinear or constrained system. One strategy is to use the EKF covariance update formula as presented in equation (10) (Rao et al., 2003).

Next we present a state estimation based approach for rapid determination of concentrations of mercury (II) and chromium (VI) from the measurement of cell population responses provided by the RT-CES.

\subsection{Concentrations estimation of chromium (VI)}

The key parameter we aim to estimate is the extracellular concentration $\left(c_{e}\right)$ of chromium (VI). This parameter is added to the toxicity equation (3) of chromium (VI) as an augmented state as follow :

$$
\begin{aligned}
\dot{c_{i}} & =k_{1}\left(k_{2} c_{e}+\frac{k_{3} c_{e}}{k_{4}+c_{e}}-c_{i}\right) \\
\dot{N} & =N\left(k_{s}-k c_{i}\right) \\
\dot{c_{e}} & =0 \quad ; \quad y=N
\end{aligned}
$$

where, $c_{i}$ is the intracellular concentration, $N$ is the cell population and $y$ is the observation. We estimate toxicant concentrations from three toxicity responses corresponding to toxicant doses $c_{e}=(0.62 ; 1.97 ; 4.25) \mu \mathrm{M}$. Note these data have not been used for modeling purpose and thus serve as cross validation data for state estimation. The results presented in Fig. (5-7) show that MHE in general has a better estimation than EKF. In addition, MHE is able to prevent an estimation of negative concentration at all time but EKF can not. It is also observed from these figures that the extracellular concentration can be correctly estimated between 10 to $15 \mathrm{hrs}$, instead of $24 \mathrm{hrs}$ as traditional the method needs. Thus a rapid estimation is achieved.
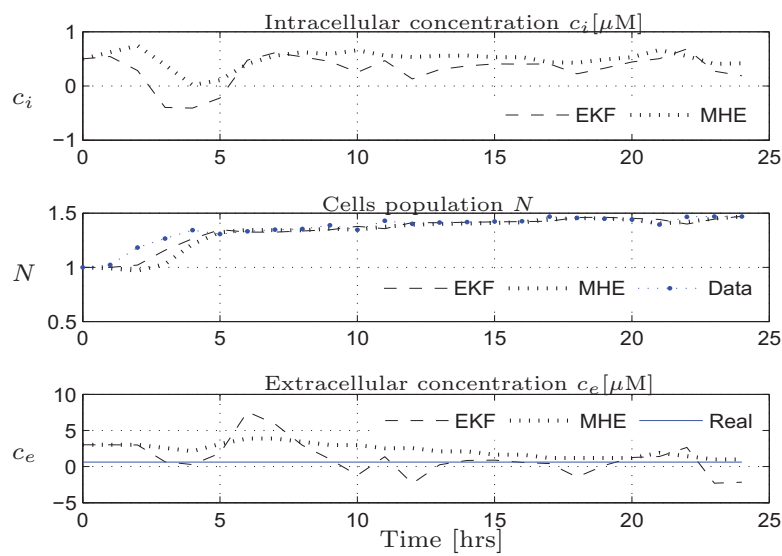

Fig. 5. Concentration estimation of chromium (VI) corresponding to real $c_{e}=0.62 \mu M$. Bottom plot : Estimation of $c_{e}$ converges to the real value $(0.62 \mu M)$ using both estimators. Top plot shows the estimation of $c_{i}$ which is not measured. The middle plot shows the estimation of cell population that is measured.

\subsection{Concentrations estimation of mercury (II) chloride}

Similar to the procedure adopted for estimating the concentration of chromium VI presented in the previous
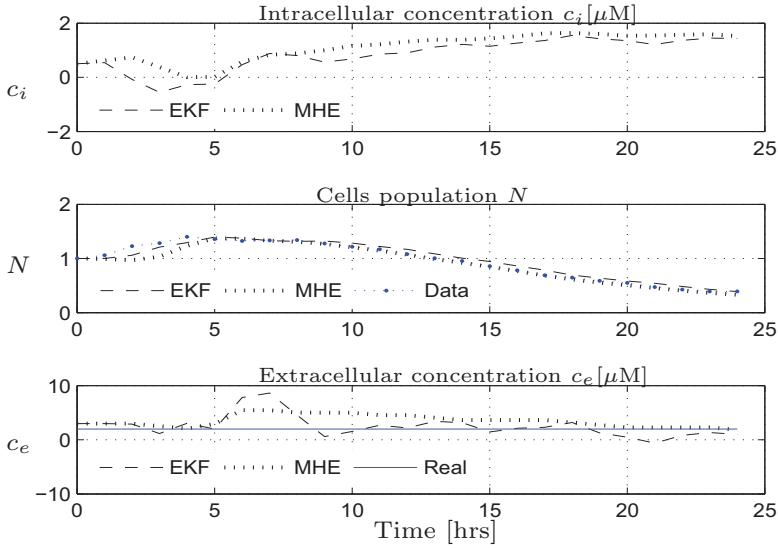

Fig. 6. Concentration estimation of chromium (VI) corresponding to real $c_{e}=1.97 \mu \mathrm{M}$. Bottom plot : Estimation of $c_{e}$ converges to the real value $(1.97 \mu M)$ using both estimators. Top plot shows the estimation of $c_{i}$ which is not measured. The middle plot shows the estimation of cell population that is measured.
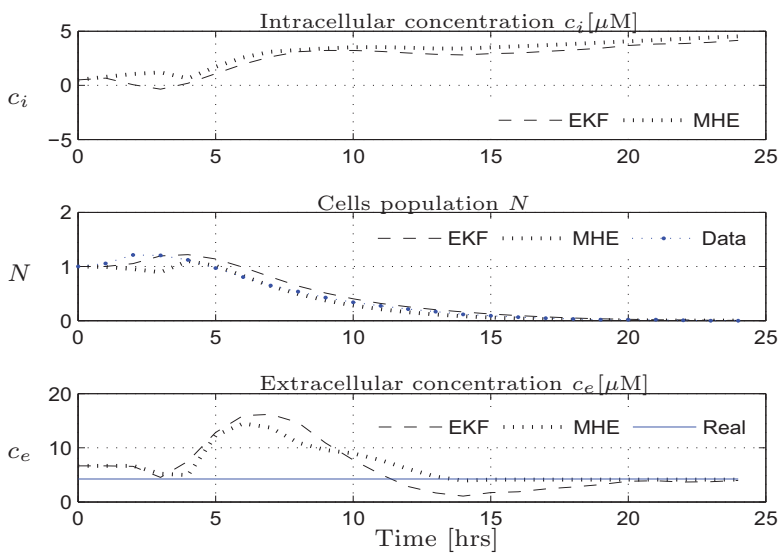

Fig. 7. Concentration estimation of chromium (VI) corresponding to real $c_{e}=4.25 \mu \mathrm{M}$. Bottom plot : Estimation of $c_{e}$ converges to the real value $(4.25 \mu \mathrm{M})$ using both estimators. Top plot shows the estimation of $c_{i}$ which is not measured. The middle plot shows the estimation of cell population that is measured.

section, we aim here to estimate the concentration of mercury (II) chloride from the available data. The concentration $c_{e}$ is added to the toxicity equation (4) of mercury (II) chloride as an augmented state similar to the augmented model (eqn. 11) for chromium (VI). We estimate the toxicant concentrations from three toxicity responses corresponding to the toxicant concentration $c_{e}=(10.43$; $22.35 ; 48.3) \mu \mathrm{M}$. The results presented in Fig. (8-10) show that both estimators (EKF and MHE) provide a good estimation of $c_{e}$ while preventing negative concentration estimation when using MHE. This shows clearly the benefits of using constraints by MHE.

Our experience shows that tuning EKF is simpler. Using MHE requires a more careful tuning of a several parameters, namely, $Q, R, Q_{-N_{h}}$, horizon length $\left(N_{h}\right)$, the constraints $w_{\min }, w_{\max }$ and also the initial conditions. In addition, the tuning may vary from different experiments. The evolution of the estimation converges by using horizon length $N_{h}=1$ for mercury (II) chloride case while at least $N_{h}=2$ is needed for chromium IV case. 
In the selection of the covariance, the $\mathrm{Q}$ matrix reflects the uncertainty of the state equations while the $\mathrm{R}$ matrix reflects the uncertainty in the measurement of CI due to other phenomena that also affect CI in addition to cell numbers.
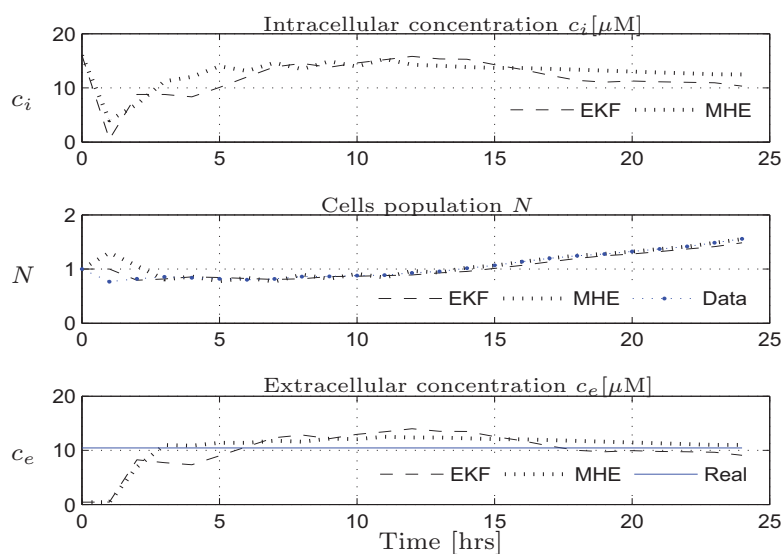

Fig. 8. Concentration estimation of mercury (II) chloride corresponding to real $c_{e}=10.43 \mu \mathrm{M}$. Bottom plot: Estimation of $c_{e}$ converges to the real value $(10.43 \mu M)$ using both estimators. Top plot shows the estimation of $c_{i}$ which is not measured. The middle plot shows the estimation of $N$ that is measured.
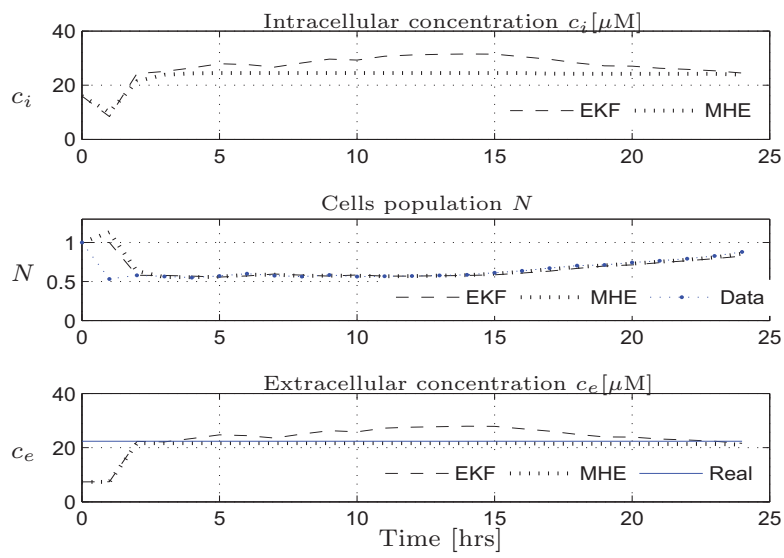

Fig. 9. Concentration estimation of mercury (II) chloride corresponding to real $c_{e}=22.35 \mu M$. Bottom plot: Estimation of $c_{e}$ converges to the real value $(22.35 \mu M)$ using both estimators. Top plot shows the estimation of $c_{i}$ which is not measured. The middle plot shows the estimation of $N$ that is measured.

\section{CONCLUSION}

An early warning system for water supply is our main goal of the work presented. This includes an early determination of the presence of specific toxicants in water by on-line estimation of their concentrations. We use mainly MHE as an on-line estimation tool in this paper. Determination of the concentration is only one of the features of the aimed early warning system. This system will also include prediction of future evolution of toxicity response using only initial measurements and prediction of cells response when the concentration of a toxicant varies. Integrating all these prediction features is the ultimate goal. Intuitively, this includes also the development of toxicity mathematical models for other kinds of common water toxicants such as sodium arsenite [As (III)] for instance.
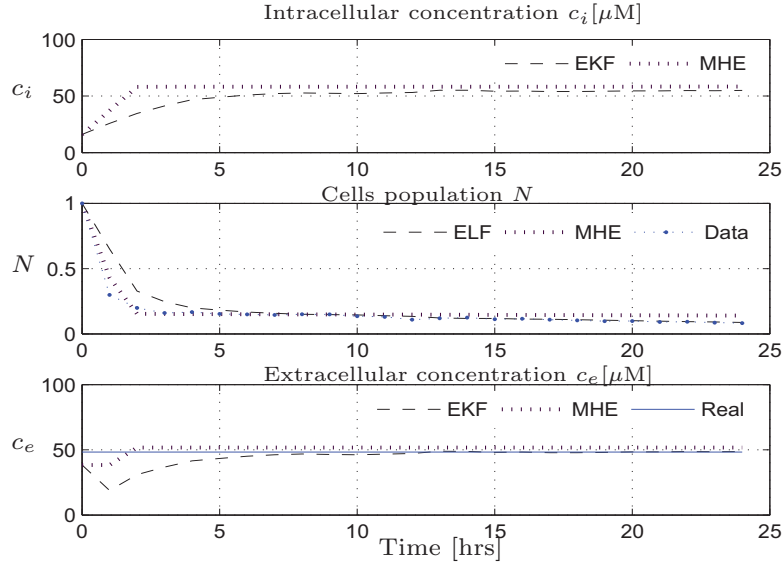

Fig. 10. Concentration estimation of mercury (II) chloride corresponding to real $c_{e}=48.3 \mu \mathrm{M}$. Bottom plot: Estimation of $c_{e}$ converges to the real value $(48.3 \mu M)$ using both estimators. Top plot shows the estimation of $c_{i}$ which is not measured. The middle plot shows the estimation of $N$ that is measured.

In addition, as has been discussed, the cell index also reflects other sophisticated physiological and pathological responses in addition to cell numbers. A model that considers other properties of the cell index will further improve on-line state estimation.

\section{ACKNOWLEDGEMENTS}

The authors gratefully acknowledge the support of the Natural Sciences and Engineering Research Council of Canada (NSERC).

\section{REFERENCES}

El-Kareh, A. and Secomb, T. (2005). Two-mechanism peak concentration model for cellular pharmacodynamics of doxorubicin. Neoplasia, 77, 705-713.

Eliaz, R., Nir, S., Marty, C., and Szoka, J. (2004). Determination and modeling of kinetics of cancer cell killing by doxorubicin and doxorubicin encapsulated in targeted liposomes. Birches. J., 64, $711-718$.

Haseltine, E. and Rawlings, J. (2005). A critical evaluation of extended kalman filtering and moving horizon estimation. Ind. Eng. Chem. Res., 44, 2451-2460.

Huang, B. and Xing, J. (2006). Dynamic modeling and prediction of cytotoxicity on microelectronic cell sensor array. Canadian Journal of Chemical Engineering, 86, 393-405.

Rao, C. and Rawlings, J. (2002). Constrained process monitoring: Moving - horizon approach. IChE Journal, 48, 97-109.

Rao, C., Rawlings, J., and Lee, J. (October 2001). Constrained linear state estimation - a moving horizon approach. Automatica, 37, 1619-1628.

Rao, C., Rawlings, J., and Mayne, D. (2003). Constrained state estimation for nonlinear discrete-time systems: stability and moving horizon approximations. Automatic Control, IEEE Transactions on, 48, 246-258.

Walter, E. and Pronzato, L. (1996). On the identifiability and distinguishability of nonlinear parametric models. Mathematics and Computers in Simulation, 42, 125-134.

Wang, Q., Kim, D., Dionysiou, D., Sorial, G., and Timberlakeb, D. (2004). Sources and remediation for mercury contamination in aquatic systems - a literature review. Environmental Pollution, $131,323-336$

Xing, J., Zhu, L., Jackson, J.A., Gabos, S., Sun, X.J., b. Wang, $\mathrm{X}$., and $\mathrm{Xu}, \mathrm{X}$. (2005). Dynamic monitoring of cytotoxicity on microelectronic sensors. Chem. Res. Toxicol, 18 (2), 154-161. 